\title{
AN INTEGRAL EQUATION FROM PHYTOLOGY
}

J. B. MILLER

(received 28 October 1963)

\section{Introduction}

We examine the equation

$$
f(\beta)=\int_{0}^{\frac{1}{2} \pi} K(\alpha, \beta) g(\alpha) d \alpha \quad\left(0 \leqq \beta \leqq \frac{1}{2} \pi\right)
$$

or, briefly,

where

$$
f=K g
$$

with

$$
K(\alpha, \beta)= \begin{cases}\cos \alpha \sin \beta & (\alpha \leqq \beta) \\ \cos \alpha \sin \beta\left\{1+\frac{1}{2} \pi(\tan \theta(\alpha, \beta)-\theta(\alpha, \beta)\}\right. & (\alpha \geqq \beta),\end{cases}
$$

This integral equation arises in connection with the problem of measuring foliage density of small plants and grasses by means of point quadrats; it is due to J. R. Philip [1]. Foliage density is defined to be the area of foliage per unit volume of space. In order to assess the foliage density within a certain spacial region, the phytologist pushes a point quadrat (which is a sharp needle, suitably mounted) through the region along a line inclined at an angle $\beta$ to the horizontal, and records the number of contacts with foliage made by the point of the quadrat per unit length of travel: this figure determines $f(\beta)$. The unknown distribution of foliage angle is given by $g: g(\alpha) d \alpha$ is the contribution to foliage density due to foliage inclined at angles between $\alpha$ and $\alpha+d \alpha$ to the horizontal (it being supposed that the foliage slopes non-preferentially to all points of the compass). The practical problem is to find $g$ from a knowledge of the values of $f(\beta)$ for a few values of $\beta$. In general the phytologist must work on che assumption that $f$ is smooth; $g$ is of course expected to be non-negative, but may be anything from constant to, say, a delta function.

The form of the kernel $K$ is due to J. Warren Wilson and J. E. Reeve [4]. $K$ is continuous over the square $\left[0, \frac{1}{2} \pi\right] \times\left[0, \frac{1}{2} \pi\right]$, but it is not symmetric: thus (1.1) is a Fredholm integral equation of the first kind whose 
$L^{2}$ theory would be covered by, say, the discussion in $[3], \S \S 3.15,3.16$. The purpose of the present note is to describe the $L^{1}$ theory, where an explicit formula for the solution $((3.5))$ can be found by quite modest means when $t$ is sufficiently smooth. The solution found is unique, but depends explicitly upon $f$ and its first three derivatives; thus to estimate $g$, many values of $f(\beta)$ are required. Consequently, the solution is principally of theoretical interest, and is unsuitable for application to experimental data. We do not examine here what further conditions on $f$ are necessary in order that $g$ be non-negative, as required.

I must thank Dr. Philip for introducing me to the subject and for the benefit of several helpful discussions, and the referee for additional comments.

\section{Range of $K$}

The transform relation (1.1) is, in more detail,

$$
\frac{1}{2} \pi f(\beta)=\frac{1}{2} \pi c_{0} \sin \beta+\sin \beta \int_{\beta}^{\frac{1}{2} \pi} g(\alpha) \cos \alpha(\tan \theta(\alpha, \beta)-\theta(\alpha, \beta)) d \alpha,
$$

where

$$
c_{0}=\int_{0}^{t \pi} g(\alpha) \cos \alpha d \alpha .
$$

In this section we shall take $K$ to be the linear operator defined by (1.1) whose domain is $L^{1}\left(0, \frac{1}{2} \pi\right)$ (briefly, $\left.L^{1}\right)$; we assume that $g$ is a function in $L^{1}$, and consider the consequent properties of its transform $t$. In this way we find necessary conditions on f for the existence of solutions $g$ in $L^{1}$.

Notice that $0 \leqq \theta(\alpha, \beta) \leqq \frac{1}{2} \pi$; for fixed $\beta \neq 0, \theta(\alpha, \beta)$ increases from 0 to $\frac{1}{2} \pi$ as $\alpha$ increases from $\beta$ to $\frac{1}{2} \pi$, while for fixed $\alpha \neq 0, \theta(\alpha, \beta)$ decreases from $\frac{1}{2} \pi$ to 0 as $\beta$ increases from 0 to $\alpha$.

Lemma 1. If $g \in L^{1}$, then

$$
\lim _{\beta \rightarrow 0} f(\beta)=\int_{0}^{\frac{1}{2} \pi} g(\alpha) \sin \alpha d \alpha, \lim _{\beta \rightarrow \frac{1}{2} \pi} f(\beta)=\int_{0}^{\frac{1}{2} \pi} g(\alpha) \cos \alpha d \alpha=c_{\alpha} .
$$

Proof. Since

$$
\tan \theta(\alpha, \beta)=\cot \beta \sqrt{\tan ^{2} \alpha-\tan ^{2} \beta},
$$

$\cos \alpha \tan \theta(\alpha, \beta)$ is an increasing function of $\alpha$, and

$$
0 \leqq \cos \alpha \tan \theta(\alpha, \beta) \leqq \cot \beta \text { for } 0<\beta \leqq \alpha \leqq \frac{1}{2} \pi \text {. }
$$

The result follows from (2.1).

Lemma 2. If $g \in L^{1}$, then $f^{\prime}(\beta)$ exists for all $\beta$ in $\left(0, \frac{1}{2} \pi\right)$, and

$$
\frac{\pi}{2} \frac{d}{d \beta}\left(\frac{f(\beta)}{\sin \beta}\right)=-\frac{1}{\sin ^{2} \beta} \int_{\beta}^{1 \pi} g(\alpha) \cos \alpha \sqrt{\tan ^{2} \alpha-\tan ^{2} \beta} d \alpha .
$$


PROOF. Formula (2.3) follows formally from (2.1) by differentiation, since

$$
\frac{\partial}{\partial \beta}(\tan \theta(\alpha, \beta)-\theta(\alpha, \beta))=-\frac{\sqrt{\tan ^{2} \alpha-\tan ^{2} \beta}}{\sin ^{2} \beta} .
$$

To prove the lemma, let $h(\beta)$ denote the righthand side of (2.3). Taking $0<\sigma<\tau<\frac{1}{2} \pi$, integrate $h(\beta)$ over $(\sigma, \tau)$, inverting the order of integration in the double integral. (This is justified by Fubini's and Tonelli's theorems, under the assumption $g \in L^{1}$.) We find

Hence

$$
\int_{\sigma}^{\tau} h(\beta) d \beta=\frac{\pi}{2} \frac{f(\tau)}{\sin \tau}-\frac{\pi}{2} \frac{f(\sigma)}{\sin \sigma} .
$$

$$
h(\tau)=\frac{\pi}{2} \frac{\partial}{\partial \tau}\left(\frac{f(\tau)}{\sin \tau}\right)
$$

for almost all $\tau$ in $\left(0, \frac{1}{2} \pi\right)$. Since in fact $h(\tau)$ exists for all $\tau$ in $\left(0, \frac{1}{2} \pi\right)$, we can assume that $f^{\prime}(\tau)$ likewise exists for all $\tau$. The result follows.

LEMMA 3. If $g \in L^{1}$, then $\lim _{\beta \rightarrow 0} f^{\prime}(\beta)=\lim _{\beta \rightarrow \frac{1}{2} \pi} f^{\prime}(\beta)=0$.

Proof. (2.3) is equivalent to

$$
\begin{array}{r}
\frac{1}{2} \pi f^{\prime}(\beta)=\frac{1}{2} \pi c_{\sigma} \cos \beta+\cos \beta \int_{\beta}^{\frac{1}{2 \pi}} g(\alpha) \cos \alpha(\tan \theta(\alpha, \beta)-\theta(\alpha, \beta)) d \alpha \\
-\operatorname{cosec} \beta \int_{\beta}^{\frac{k}{k} \pi} g(\alpha) \cos \alpha \sqrt{\tan ^{2} \alpha-\tan ^{2} \beta} d \alpha .
\end{array}
$$

The value of the limit as $\beta \rightarrow \frac{1}{2} \pi$ follows without difficulty. To derive the limit as $\beta \rightarrow 0$, one first shows that

$$
\lim _{\beta \rightarrow 0} \int_{\beta}^{\frac{1}{2} \pi} g(\alpha) \cos \alpha \theta(\alpha, \beta) d \alpha=\frac{1}{2} \pi c_{\theta},
$$

and then uses (2.5). We omit the details.

LEMMA 4. If $g \in L^{1}$, then $f^{\prime \prime}(\beta)$ exists for almost all $\beta$ in $\left(0, \frac{1}{2} \pi\right)$, determining a measurable function, and for such $\beta$,

$$
\frac{1}{2} \pi \cos ^{3} \beta\left(f(\beta)+f^{\prime \prime}(\beta)\right)=\int_{\beta}^{\frac{1}{2} \pi} \frac{g(\alpha) \cos \alpha d \alpha}{\sqrt{\tan ^{2} \alpha-\tan ^{2} \beta}} .
$$

The proof follows closely that of Lemma 2 , so we omit it.

We conclude from Lemmas 2, 3 and 4 that the range of $K$ is contained in the class of functions $f$ which are defined and have absolutely continuous first derivative on the open interval $\left(0, \frac{1}{2} \pi\right)$, with $f^{\prime}(0+0)=f^{\prime}\left(\frac{1}{2} \pi-0\right)=0$.

It can be shown that $f$ has a third derivative if $g$ is also absolutely continuous and satisfies certain integrability conditions. 


\section{Solution of the integral equation}

The solution in $L^{1}$ is unique.

THEOREM 1. The integral equation (1.1) has at most one solution $g$ in $L^{1}$, if $f$ is given.

Proof. Let $g_{1}$ and $g_{2}$ be two solutions of (2.1), in $L^{1}$. By Lemma 2,

$$
\int_{\beta}^{\frac{1}{2} \pi}\left(g_{1}(\alpha)-g_{2}(\alpha)\right) \cos \alpha \sqrt{\tan ^{2} \alpha-\tan ^{2} \beta} d \alpha=0
$$

for all $\beta$ in $\left(0, \frac{1}{2} \pi\right)$. Make the change to variables $x$ and $y$ defined by

$$
\tan ^{2} \alpha=x, \tan ^{2} \beta=y,
$$

and write

(3.1) becomes

$$
r(x)=\frac{h\left(\tan ^{-1} \sqrt{ } x\right)}{2 x^{\frac{1}{2}}(1+x)^{\frac{3}{2}}}, \quad h(\alpha)=g_{1}(\alpha)-g_{2}(\alpha) ;
$$

$$
\int_{y}^{\infty}(x-y)^{\frac{1}{2}} r(x) d x=0 \text { for all } y \text { in }(0, \infty) .
$$

Titchmarsh's convolution theorem ${ }^{1}$ implies that $r(x)=0$ for almost all $x$. The result follows.

In $\S 2$ we have found necessary conditions on $f$ for the existence of a solution $g \in L^{1}$. We now find sufficient conditions, and obtain an explicit formula for the solution. Formally, this is done as follows. The solution $g$ satisfies the differentiated form (2.3); change to the variables $x$ and $y$ of (3.2), and introduce functions $p$ and $q$ by writing

$$
\begin{array}{ll}
p(y)=-\frac{\pi}{2} \sin ^{2} \beta \frac{d}{d \beta}\left(\frac{f(\beta)}{\sin \beta}\right) & \left(0<\beta<\frac{1}{2} \pi ; 0<y<\infty\right), \\
q(x)=\frac{g(\alpha)}{2 \tan \alpha \sec ^{3} \alpha} & \left(\beta<\alpha<\frac{1}{2} \pi ; y<x<\infty\right) .
\end{array}
$$

Equations (2.3) becomes

$$
p(y)=\int_{y}^{\infty}(x-y)^{\frac{1}{2}} q(x) d x .
$$

Then

so that

$$
\begin{aligned}
\int_{t}^{\infty} \frac{(y-t)^{-\frac{1}{2}}}{y^{2}} p(y) d y & =\int_{t}^{\infty} q(x) d x \int_{t}^{x} \frac{(x-y)^{\frac{1}{2}}(y-t)^{-\frac{1}{2}}}{y^{2}} d y \\
& =\int_{t}^{\infty} q(x) \frac{(x-t)}{x^{\frac{1}{2}} t^{\frac{1}{2}}} \frac{\pi}{2} d x
\end{aligned}
$$

1 [2], p. 325, Theorem 152. 
Therefore

$$
\frac{2 t}{\pi} \int_{t}^{\infty} \frac{(y-t)^{-1}}{y^{2}} p(y) d y=\int_{t}^{\infty} d u \int_{u}^{\infty} \frac{q(x)}{x^{t}} d x
$$

i.e.

$$
\begin{aligned}
(-1)^{2} \frac{q(t)}{t^{1}} & =\frac{2}{\pi} \frac{d^{2}}{d t^{2}}\left(t \frac{t^{2}}{\int_{t}^{\infty}} \frac{(y-t)^{-\frac{t}{2}}}{y^{2}} p(y) d y\right) \\
& =\frac{2}{\pi} \frac{d^{2}}{d t^{2}} \int_{1}^{\infty} \frac{(u-1)^{-\frac{1}{2}}}{u^{2}} p(t u) d u \\
& =\frac{2}{\pi} \int_{1}^{\infty}(u-1)^{-\frac{1}{2}} p^{\prime \prime}(t u) d u
\end{aligned}
$$

$$
q(t)=\frac{2}{\pi} \int_{t}^{\infty}(y-t)^{-\frac{1}{t}} p^{\prime \prime}(y) d y .
$$

In terms of the original functions, this is

$$
g(\alpha)=\tan \alpha \sec ^{3} \alpha \int_{\alpha}^{\frac{t^{\pi}}{2}} \frac{3 \cos ^{2} \tau \sin \tau\left(f(\tau)+f^{\prime \prime}(\tau)\right)-\cos ^{3} \tau\left(f^{\prime}(\tau)+f^{\prime \prime \prime \prime}(\tau)\right)}{\sqrt{\tan ^{2} \tau-\tan ^{2} \alpha}} d \tau
$$

Rather than justify the above argument, it is simpler to start with (3.5), and show that it defines a solution of (2.1) under suitable conditions on $f$. If this is done (and we shall not elaborate the details here), we obtain

THEOREM 2. Let $f$ be such that $f^{\prime \prime}$ exists and is absolutely continuous on $\left[0, \frac{1}{2} \pi\right]$, and

$$
f^{\prime}(0)=f^{\prime}\left(\frac{1}{2} \pi\right)=0 .
$$

Then $g(\alpha)$, given by (3.5), exists for almost all $\alpha$, and $g$ is the solution of (1.1) belonging to $L^{1}$.

Finally, to round off the discussion, we consider the circumstances in which a solution $g$ of (1.1) can be found by solving one of the differentiated forms of the equation, (2.3) or (2.6). It is evident, for example, that a solution $g$ of (2.3), which does not contain the constant $c_{g}$ depending upon the solution, may not be a solution of (2.1). We state without proof

ThEOREM 3. (i) Let $f^{\prime}$ be absolutely contimuous on $\left(0, \frac{1}{2} \pi\right)$. If $g$ is a solution in $L^{1}$ of (2.6), it is also a solution of (2.3) if and only if $f^{\prime}\left(\frac{1}{2} \pi-0\right)=0$.

(ii) Suppose instead that $f$ is absolutely continuous on $\left(0, \frac{1}{2} \pi\right)$. If $g$ is a solution in $L^{1}$ of $(2.3)$, it is also a solution of $(2.1)$ if and only if $c_{s}=f\left(\frac{1}{2} \pi-0\right)$.

\section{References}

[1] Philip, J. R., Analysis of the spatial distribution of foliage by point quadrats at several inclinations (to be submitted to the Aust. J. of Dotany). 
[2] Titchmarsh, E. C., Introduction to the theory of Fourier integrals. Oxford U.P., 1937.

[3] Tricomi, F. G., Integral equations. Interscience, New York, 1957.

[4] Warren Wilson, J., Inclined point quadrats, with Appendix by J. E. Reeve. The New Phytologist 59 (1960), 1-8.

\section{Australian National University,} Canberra. 